\title{
Sialidosis type 1: cherry red spot-myoclonus syndrome with sialidase deficiency and altered electrophoretic mobilities of some enzymes known to be glycoproteins
}

\section{Clinical findings}

\author{
P. K. THOMAS, J. D. A B R A M , D A L L A S S A L L O W,
} A N D G. S T E W A R T

From the Departments of Neurological Science and Ophthalmology, Royal Free Hospital, the MRC Human Biochemical Genetics Unit, Galton Laboratory, University College, and the Department of Psychological Medicine, St Bartholomew's Hospital, London

SUMMARY A family is described with three affected brothers, two of whom were examined, born to consanguineous parents, who in early adult life began to experience ataxia, intention myoclonus, and progressive visual failure. The brothers examined had cherry red spots at the maculae and cataracts. They were of normal intelligence. The intention myoclonus responded partially to treatment with clonazepam and pheneturide, but not to 5-hydroxytryptophan in combination with carbidopa or to sodium valproate. Studies in one patient showed the excretion of large quantities of sialylated oligosaccharides in the urine. Both patients showed deficient sialidase activity in their cultured fibroblasts. Further studies on cultured skin fibroblasts revealed increased electrophoretic mobility of six glycoprotein enzymes that was returned approximately to normal by treatment with sialidase. The clinical and biochemical findings indicate that these patients are further cases of the newly described condition sialidosis type 1 .

In 1976, two members of a family presented with an unusual neurological disorder consisting of dysarthria, limb ataxia, and intention myoclonus. Both were of normal intelligence. They were found to have cherry red spots at their maculae and perinuclear cataracts. The presence of the cherry red spots suggested a lysosomal storage disorder. Investigations on leucocytes and cultured skin fibroblasts demonstrated abnormalities in the electrophoretic mobility of a number of glycoprotein enzymes that were returned approximately to normal by treatment with sialidase (neuraminidase). At about the same time, O'Brien and Rapin (1977) reported their observations on two sisters with the "cherry red spot-mycclonus syndrome" (Rapin et al., 1975) in whom they found large quantities of sialylated oligosaccharide in the

Address for correspondence and reprint requests: Professor P. K. Thomas, Royal Free Hospital, Pond Street, London NW3 2QG.

Accepted 27 March 1979 urine. Studies on cultured skin fibroblasts demonstrated a deficiency of an acid sialidase (O'Brien, 1977) which is probably the primary defect in these patients (O'Brien, 1978). Their results have been documented in greater detail by O'Brien (1978) and Rapin et al. (1978). G. H. Thomas et al. (1978) also reported a case of the cherry red spot-myoclonus syndrome in whom a deficiency of sialidase was detected. Durand et al. (1977) provided clinical descriptions of two siblings from a family De $P$ included in Strecker et al. (1976, 1977). These patients had been shown by Strecker et al. (1976) to excrete sialylated cligosaccharides and to have deficient $\alpha$-neuraminidase (sialidase) activity in their leucocytes. The two affected individuals had cherry red spots and punctate lens opacities but no other marked clinical features, in particular, no intention myoclonus. Itoyama et al. (1978) described members of a family with progressive ataxia, intention myoclonus, and cherry red spots without studies on sialidase but 
demonstrating the presence of sialylated glycopeptides in their patients' urine.

Further studies on our patients established that urine contained large amounts of sialylated oligosaccharides and that there was a deficiency of sialidase in their cultured skin fibroblasts. The present communication documents their clinical features in comparison with those of the cases described by Durand et al. (1977), Itoyama et al. (1978), Rapin et al. (1978), and G. H. Thomas et al. (1978). The biochemical changes will be presented in greater detail elsewhere (Swallow et al., 1979) but we may note that the electrophoretic investigations have produced the same result when applied to cultured skin fibroblasts from three of O'Brien's patients (Swallow, in preparation).

\section{Case reports}

The family (Fig. 1) was of Iranian origin. Three male siblings born to first cousin parents were affected, two of whom were examined and investigated. A sister, who was not examined, was stated to be unaffected, the same being true of their parents. Facial appearance and body habit were normal in the two brothers examined and reported to be normal in the rest of the family.

\section{CASE IV.1}

This man aged 31 years when first seen, was normal until the age of 19 or 20 years, when he became unsteady on his feet. Incoordination of his hands appeared about a year later, and at the same time his speech became abnormal. His speech was normal if he spoke slowly, but attempts at rapid speech were disturbed by jerky interruptions. There was also slight dysphagia. The involuntary movements were worse if he was excited, and they had been observed to occur during sleep. Some visual deterioration had been noted at the age of 27 years. His condition had deteriorated slowly since the time of onset.
Examination revealed an individual with normal intelligence. His speech was explosive and interrupted by jerky sounds that occurred both during expiration and inspiration. There was action myoclonus of the facial muscles. Upward gaze was slightly defective. There was no nystagmus. Visual acuity was $6 / 36$ in both eyes and the visual fields were full. Perinuclear lens opacities were present bilaterally (Fig. 2). The optic discs were normal but bilateral early cherry red spots were detected (Fig. 3).

There was ataxia in all four limbs. His gait was broad-based and unsteady. Movements were interrupted by abrupt myoclonic jerks, especially in the upper limbs. Power and tone were normal as were his tendon reflexes, and both plantar responses were flexor. There was no sensory loss. Examination of other systems was negative; blood pressure was $120 / 80 \mathrm{mmHg}$. There was no hepatic or splenic enlargement.

\section{CASE IV.2}

This man aged 28 years, was stated to be similarly affected with an onset early in the third decade. He had been investigated in Japan, but no detailso as to the diagnosis reached were known.

\section{CASE IV.3}

This man aged 26 years, was normal until the age of 20 years. He had been a keen athlete. At thato age he developed tremor of his hands, legs, and head, and speech became difficult. There waso slight dysphagia. His disability had slowly: worsened. He was aware of involuntary muscle jerking which was increased by cigarette smoking. He stated that these movements ceased during intercourse, and after orgasm remained in abeyance for some hours. He had noticed no visual difficulty.

Examination revealed normal intelligence. There was gross dysarthria of cerebellar type. He showed sudden contractions of the facial muscles

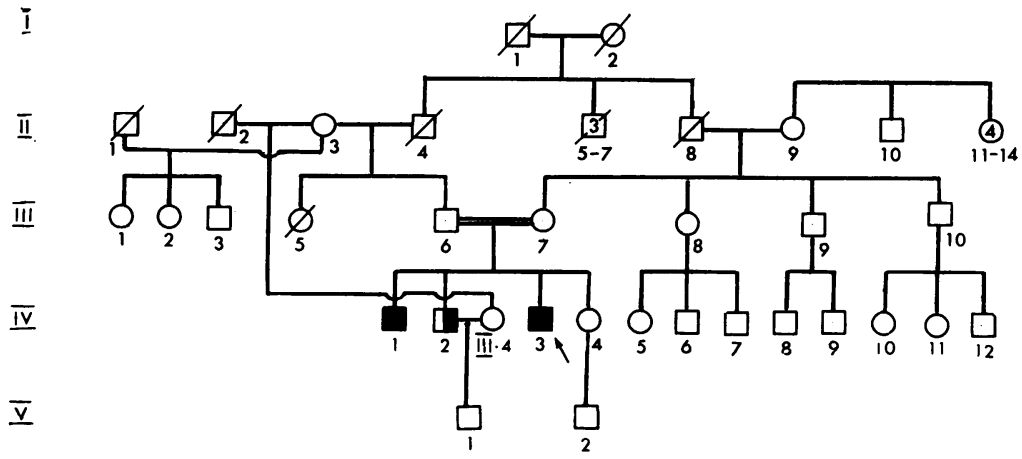

Fig. 1 Pedigree of family. Filled symbols indicate affected individuals, the half-filled symbol an individual presumed to be affected, and open symbols unaffected individuals. Death is denoted by a line through the symbol, the propositus by the arrow. 


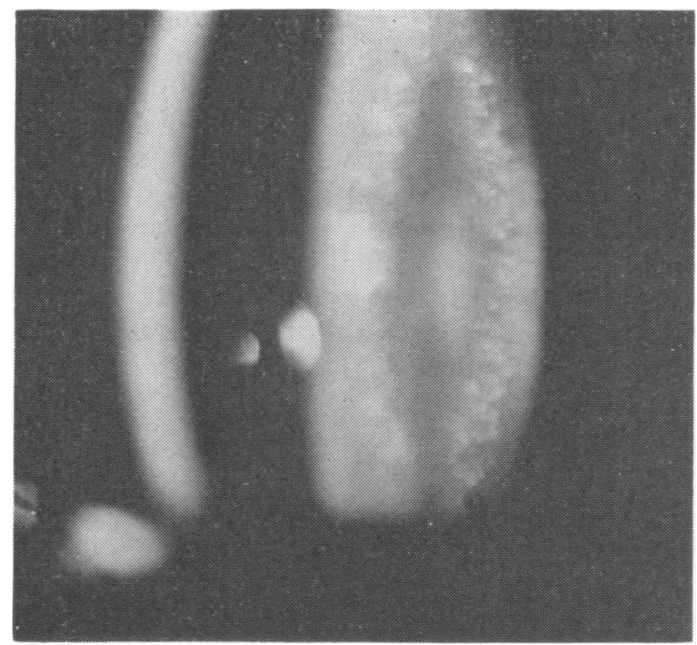

Fig. 2 Slit lamp photograph showing perinuclear lens opacities in case IV.I.

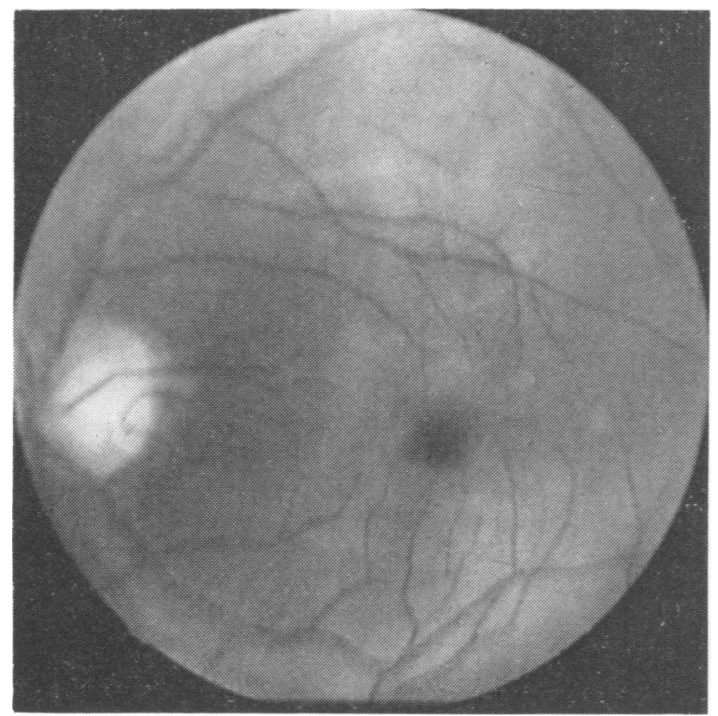

Fig. 3 Fundal photograph from case IV.1. showing early macular cherry red spot.

on facial movement. His visual acuity was normal, but on testing his visual fields there were bilateral pericentral scotomata. A typical cherry red spot was present at the macula on both sides (Fig. 4), the surrounding retina being slightly heaped-up and pale. Perinuclear lenticular opacities were evident (Fig. 5) but less obvious than in his brother. There was severe ataxia in the upper limbs with superimposed myoclonic jerks and less severe changes in the lower limbs. Power and tone were normal, as were the tendon reflexes. The

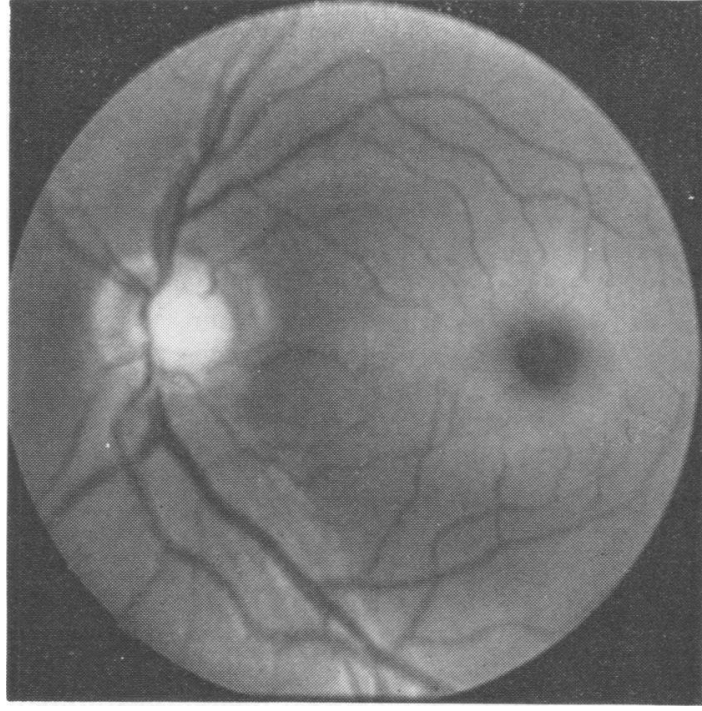

Fig. 4 Fundal photograph from case IV.3 showing macular cherry red spot, the surrounding retina being heaped-up and pale.

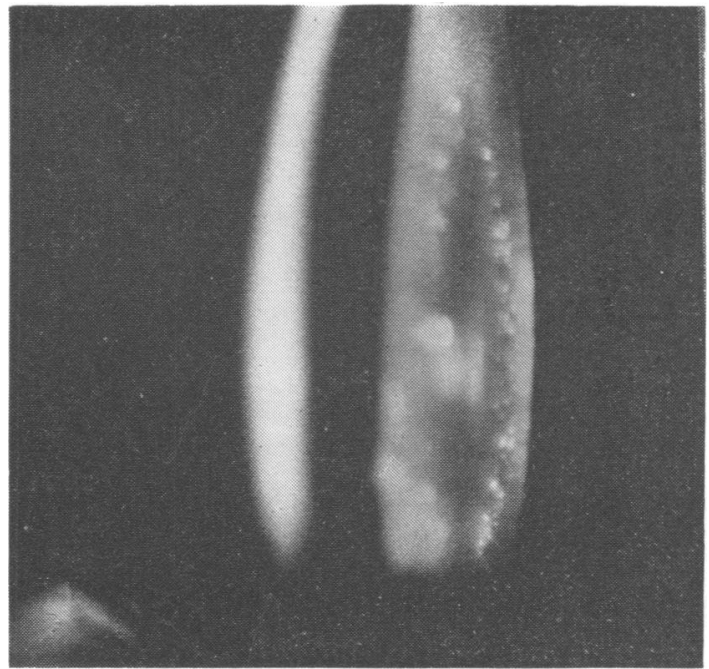

Fig. 5 Slit lamp photograph showing perinuclear lens opacities in case IV.3.

plantar responses were flexor and sensation intact. Examination of other systems was negative; blood pressure was $110 / 70 \mathrm{mmHg}$.

\section{INVESTIGATIONS AND TREATMENT}

Routine haematological and biochemical investigations were normal in both brothers, with normal serum caeruloplasmin and lipoprotein levels. A 
skeletal survey was normal. The ECG revealed no abnormalities.

The EEG was abnormal in both brothers with an excess of $16-26 \mathrm{~Hz}$ fast activity, possibly attributable to medication (clonazepam). There were frequent ill-formed generalised paroxysmal features and a poverty of normal rhythmic activity. Hyperventilation and photic stimulation produced no noteworthy changes. The abnormalities were greater in the elder brother.

The electroretinogram (ERG) was of normal amplitude, latency, and waveform (Fig. 6, upper traces). The visual evoked responses (VER) to a single flash stimulus showed a poorly defined, low amplitude negative component in the younger brother at $100 \mathrm{~ms}$, with a higher amplitude positive component at $130 \mathrm{~ms}$. The potentials were poorly defined in the elder brother (Fig. 6, middle traces). Comparable results were obtained for a patterned stimulus. The somatosensory responses, recorded from the left parietal region on stimulating the right median nerve at the wrist (Fig. 6, lower traces), showed early components (in the first $50 \mathrm{~ms}$ ) of normal latency but with grossly enlarged amplitudes, especially the positive component at $30 \mathrm{~ms}(\overline{\mathbf{P} 30})$.

ERG

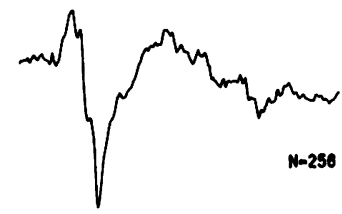

VEP

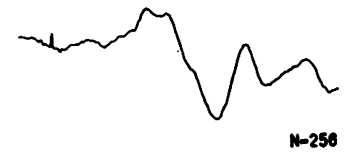

SEP

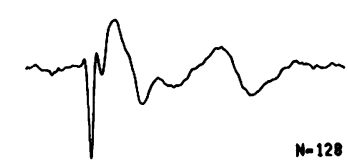

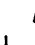<smiles>[13CH3]</smiles>

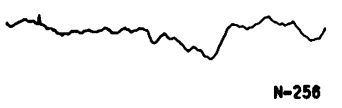

$\mathrm{I}_{+}^{-}$

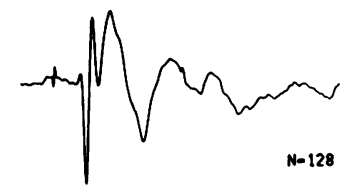

Both brothers were treated with clonazepam at a total dosage of $3 \mathrm{mg}$ per day and showed a moderate improvement in the severity of the myoclonus. Higher dosages were not tolerated because of drowsiness. The elder brother experienced a single major seizure after inadvertently ceasing this preparation. 5-Hydroxytryptophan (5-HTP) was also administered at a maximal dosage of $1.2 \mathrm{~g}$ per day in combination with 100 $\mathrm{mg}$ carbidopa per day with little noticeable effect. The younger brother was given sodium valproate at a dosage of $600 \mathrm{mg}$ per day without benefit and pheneturide at a dosage of $1 \mathrm{~g}$ per day with slight improvement.

\section{Biochemical studies}

\section{URINARY OLIGOSACCHARIDES}

Urine from one brother only was examined. Using the methods of O'Brien (1977), the urine was shown to contain sialylated oligosaccharides composed of the same sugars demonstrated by Strecker et al. (1977) in urinary oligosaccharides from their cases of sialidase deficiency but no other sugars (that is, galactose, mannose, and glucosamine, bus no fucose or glucose, or amino-acids). Free $\mathrm{N} \rightleftharpoons \vec{D}$

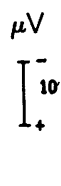

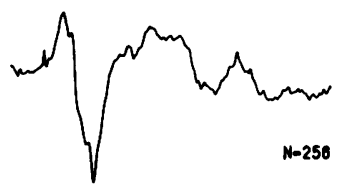
$N-128$

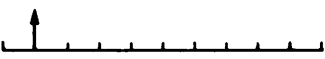

Fig. 6 Recordings of electroretinogram (ERG), visual evoked potentials ( $V E P$ ), and somatosensory evoked potentials (SEP) from case IV.3 (on left) and case IV.1 (on right). The ERG was recorded from a non-corneal electrode placed on the bridge of the nose with stimulation of both eyes with brief flashes, and averaged simultaneously with the VEP recorded from the mid-occipital region. The SEP was recorded from the left parietal region with stimulation of the right median nerve at the wrist. Stimulus at arrow, time marker $25 \mathrm{~ms}$, calibrations in $\mu V$. 
acetyl neuraminic acid was demonstrated by thiobarbituric acid assay after mild hydrolysis of the isolated oligosaccharide.

\section{SIALIDASE ASSAY}

Fibroblasts from both brothers were found to have reduced sialidase activity compared with normal control subjects using fetuin and 2-(3' methyoxyphenyl)-N-acetyl-D neuraminic acid as substrate; the deficiency was more marked using the methoxyphenyl substrate (see Swallow et al. (1979) for table).

\section{OTHER ENZYME STUDIES AND ELECTROPHORETIC} STUDIES

These are described in detail by Swallow et al. (1979). Quantitative studies showed normal levels of acid phosphatase, $\alpha$-galactosidase, $\beta$-galactosidase, $\beta$-glucuronidase, and $\mathrm{N}$-acetyl- $\beta$-D-hexosaminidase in leucocytes, plasma and/or fibroblasts as compared with controls. Electrophoresis revealed increased anodal mobility in the isozymes of $\alpha$-fucosidase, acid phosphatase, $\mathrm{N}$-acetyl- $\beta$-Dhexosaminidase, $\alpha$-mannosidase, $\alpha$-galactosidase, and adenosine deaminase. All are known to be lysosomal apart from adenosine deaminase. Several known glycoprotein enzymes were unaffected $(\beta$-galactosidase, $\beta$-glucuronidase and esterase $\mathrm{A}_{4}$ ) as were plasma glycoproteins. Treatment of cell extracts with sialidase of bacterial origin returned the unusual anodal mobility approximately to normal.

\section{Discussion}

The two cases described here displayed an unusual disorder, with an onset at the beginning of the third decade of life, characterised by ataxia, intention myoclonus, cherry red spots at the maculae, and cataracts. Neither had any abnormality of facial appearance or body habit.

Urine was examined from one of the cases and found to contain large amounts of sialylated oligosaccharide. Both patients showed evidence of sialidase deficiency in cultured skin fibroblasts. Details of these investigations and the finding of altered electrophoretic mobility in several enzymes known to be glycoproteins are given in another paper (Swallow et al., 1979) where it is suggested that the altered enzymes, which may be restored approximately to normality with bacterial sialidase, could serve as the basis for an easier test than sialidase assay until a suitable substrate is commercially available. Quantitative assay of other enzymes, in particular $\beta$-galactosidase, was normal in body fluids and cells.
The literature contains recent reports of a number of cases of sialylated oligosacchariduria and sialidase deficiency with cherry red spots, myoclonus, and normal intelligence, and also cases which have in addition short stature, bony abnormalities, and frequent intellectual deficit. A recent review by Lowden and O'Brien (1979) surveys these and attempts to establish two categories, type 1 sialidosis, where the patients have a normal body habit, cherry red spots, myoclonus, and nearly always normal levels of $\beta$-galactosidase, and type 2 , in which patients have short stature, bony abnormalities, and often deficient $\beta$-galactosidase in tissues and body fluids. Their review takes into account some reports before the time when the occurrence of sialylated oligosaccharides in the urine and sialidase deficiency in cells from patients with cherry red spots at the macula and myoclonus were recognised. However, all such patients so far tested have shown these biochemical features. A recently described family with cherry red spots and progressive cerebellar ataxia with a juvenile onset (Johnson et al., 1977) does not show myoclonus and has a deficiency of hexosaminidase $\mathbf{B}$ in the affected individuals and a partial deficiency in heterozygotes (Johnson and Chutorian, 1978). It thus represents a distinct entity and is a variant of Tay-Sachs' disease.

The family described here corresponds clinically and biochemically to the type 1 sialidosis of Lowden and O'Brien. The basis of Lowden and O'Brien's type 1 sialidosis is a group of five cases, three described by Rapin et al. (1978), one by G. H. Thomas et al. (1978), and one by Goldstein et al. (1974) which was reinvestigated by O'Brien and Rapin (1977). All have been shown to excrete large quantities of sialylated oligosaccharides in the urine and the first four have sialidase deficiency in cultured skin fibroblasts (O'Brien and Rapin, 1977; O’Brien, 1978; G. H. Thomas et al., 1978). The two cases described here and three cases of O'Brien have been shown to have the same electrophoretic abnormality of enzymes known to be glycoproteins (Swallow et al., 1979; Swallow, in preparation) but this property has not been investigated in sialidosis type 2 .

Cases with biochemical confirmation have also been presented by Durand et al. (1977) and Justice et al. (1977). The family De P of Durand et al. is unusual in that the two affected individuals at the age of 22 and 9 years had cherry red spots and dyschromatopsia with punctate lenticular opacities but no other marked neurological features. The paper of Justice et al. (1977) concentrates on enzymatic findings: $\beta$-galactosidase was less than $10 \%$ in white cells and fibroblasts 
(Wenger et al., 1978), and this case is better regarded as type 2 sialidosis or an intermediate form.

The patient's parents and children reported by G. H. Thomas et al. (1978) were shown to have partial deficiency of sialidase, as did the parents of the two sisters described by Rapin et al. (1978) and O'Brien (1978). This confirmed that the defect is inherited as an autosomal recessive condition and that the sialidase deficiency is the primary defect in these patients.

A further study has been described with necropsy findings in one of two affected brothers born to consanguineous parents by Itoyama et al. (1978), although sialidase activity was not tested. Sialylated glycopeptides were recovered from their urines and $\beta$-galactosidase assays were normal.

\section{CLINICAL FEATURES}

Apart from the family De $P$ of Durand et al. (1977) the dominant clinical feature in all cases has been intention myoclonus. In our two patients, speech was dysarthric and disturbed by involuntary jerks affecting the respiratory and articulatory muscles. Coordination of the limbs was poor and interrupted by myoclonic jerks. In Case IV.1, the jerks of the lower limbs and trunk were sufficiently violent to throw him to the ground. He was, therefore, unable to walk without support.

The jerks could be provoked not only by active movement, but also by passive joint movement, tactile stimuli, and sudden loud noise. Our patient also displayed a regular intention tremor, particularly in the upper limbs, suggesting a cerebellar deficit. This was noted in the cases reported by G. H. Thomas et al. (1978) and Itoyama et al. (1978), and nystagmus was observed in two of the patients documented by Rapin et al. (1978); the younger patient in the family De $\mathrm{P}$ of Durand $e t$ al. (1977) had mild motor incoordination.

Our first case had a single major seizure, occasioned by precipitate anticonvulsant withdrawal, but otherwise generalised seizures were not a feature in this family. Case 3 of Rapin et al. had two major seizures during adolescence and case 1 recurrent generalised seizures from the age of 11 years. The cases of Itoyama et al. (1978) also had generalised seizures.

Somatosensory evoked potentials in both of the cases recorded here displayed the exaggerated early components, particularly the early positive wave ( $\overline{\mathbf{P} 30})$, typical of myoclonus (Dawson, 1947). This was found by Engel et al. (1977), who also mentioned the occurrence of positive spikes at the vertex in the EEG coincident with the myoclonus. The EEG in our cases showed only minor paroxysmal changes.

The myoclonic jerks have consistently been found difficult to control. Conventional anticonvulsants have had little effect, although clonazepam and pheneturide in our patients produced a modest improvement. Oral 5-HTP in combination with carbidopa gave rise to little noticeable effect. In case 1 of Rapin et al., this combination reduced the frequency of the myoclonus but resulted in no functional benefit. In case 3 , there was a more dramatic improvement, but this was associated with a manic state and with prolonged depression on withdrawal. Itoyama et al. (1978) reported benefit from nitrazepam.

Cherry red spots at the maculae have been a consistent feature, although it is of interest that in one of the patients of Rapin et al., the cherry red spots, first noticed at the age of 8 , faded when she was aged 19 years. The two affected individuals of the De $\mathrm{P}$ family (Durand et al., 1977) presented with failing vision. The cherry red areas have been noted to be surrounded by a halo of heaped-up greyish or whitish retina in some instances. In our case IV.3, they were associated with bilateral pericentral scotomata. The opti 0 discs have been consistently normal. Both of ou $\omega$ cases showed perinuclear cataracts, and this was 웅 probably the main cause of the reduced visuan $د$ acuity in case IV.1. The case of G. H. Thomas et al. showed punctate opacities in the anteriog and posterior subcapsular regions, and case 1 de Rapin et al. displayed "white flakes in the adu岱 nucleus of the lens reminiscent of those seen i $\overrightarrow{0}$ myotonic dystrophy." Itoyama et al. (1978) showed ฮै "mild lens turbidity on slit lamp examination" in their cases. The older affected member of the De P family (Durand et al., 1977) showed punctate lenticular opacities. The ERG was examined in case 1 of Rapin et al. and found to be normal, as it was in the patient of G. H. Thomas et al. and in our two cases. The low VER amplitudes found in our two cases and in case 1 of Rapin et al. are probably not just the result of reduced visual acuity as this was normal in our case IV.3.

Intelligence has been normal in all cases with the exception of case 2 of Rapin et al. who had mild mental retardation, first noticed at the age of 5 years, and the patient of G. H. Thomas et al. who showed "possible dyscalculia and memory deficit." The elder brother in this case, presumed to have the same disorder, showed progressive intellectual deterioration from the age of 7 years. He was also unusual in that he developed progressive loss of "physical strength" from that age, became bedridden at the age of 32 years because of "spastic weakness", and died at the age of 33 
years with uncontrollable seizure activity. His brother showed mild proximal weakness, but otherwise weakness has not been evident. All cases have been noted to have had increased tendon reflexes apart from our two patients, although the plantar responses have been flexor throughout. No sensory loss has been detected.

Cases 1 and 2 of Rapin et al. developed scoliosis but, apart from this, bony abnormalities have not been detected. It is of some interest that both of these cases started to complain in late childhood of severe pains in the hands and feet during hot weather. These are reminiscent of the pains that may occur in Fabry's disease. Cutaneous changes have not occurred and organomegaly has not been detected, apart from moderate hepatomegaly in the younger member of the family De P (Durand et al., 1977).

The cases exhibit variation in their clinical features which suggests a degree of genetic heterogeneity. While cherry red spots were first noted at the age of 8 years at a routine ophthalmological examination to be followed by myoclonus at the age of 16 years in patient 3 of Rapin et al. (1978), the cases of Durand et al. (1977) did not at the time of publication show any myoclonus. The elder, a man, whose age is given as 32 years, presented with a history of visual failure at the age of 15 years and the younger, a female, whose age is given as 13 years 8 months, had poor vision from the beginning of primary school. Their general similarity to the other cases, their hyperreflexia, shared by all reported cases except those described here, the mild motor incoordination of the younger patient, and the common findings on urine examination and enzyme assay warrant their inclusion in this clinical group. However, we note that Strecker et al. (1976) held that their hydrolytic enzymes were not unusually sialylated which would imply a distinction between them, our cases, and those of O'Brien which we have tested (Swallow, unpublished). The genetic heterogeneity may be examined further by comparing ages of onset (Haldane, 1941). This varies from the first to the third decade; the rate of progress of the disease also varies, being in general more rapid in the cases with the earlier onset.

In the family described here the onset has been in the third decade and the progress relatively slow. In the family described by Itoyama et al. (1978) the age of onset was in the early years of the second decade and the progress was to gross incapacity by the end of the decade, one patient dying at the age of 18 years. The consistency within the two families and the difference between these families indicates different abnormal genes in each family. Unfortunately the progeny affected in both families are all male and in neither family do we have observations on presumptive heterozygotes. While the possibility of sex-linked recessive inheritance exists in these two families, their consanguinity argues for autosomal recessive inheritance. The family De $P$ of Durand et al. (1977) is also consanguineous, and the affected individuals represent both sexes. The studies on other patients' relatives (O'Brien, 1978; G. H. Thomas et al., 1978) confirm autosomal recessive inheritance in them. It appears likely that all cases have the same mode of inheritance and that the variation is potentially allelic although different loci cannot be excluded at present. In the consanguineous families, if they are autosomal recessive, the affected individuals are truly homozygous for the same mutant gene, and differences between the families may result from different mutant alleles at the same locus.

The relationship between the group of patients discussed here and patients with other clinical pictures and sialidase deficiency is unclear. It is treated in the review of Lowden and O'Brien (1979) and need not be enlarged upon here. However, we believe that the present group-that is, sialidosis 1-has sufficient internal consistency to constitute a clinical entity although it contains within itself evidence of genetic heterogeneity.

We wish to thank Dr C. D. Calnan for referring the cases and the Friedreich's Ataxia Group for financial support. We are also indebted to Dr Ann Harden, Dr G. Pampiglione, and Dr B. B. MacGillivray for the electrophysiological studies.

\section{References}

Dawson, G. D. (1947). Investigations on a patient subject to myoclonic seizures after sensory stimulation. Journal of Neurology, Neurosurgery, and Psychiatry, 10, 141-162.

Durand, P., Gatti, R., Cavalieri, S., Borrone, C., Tondeur, M., Michalski, J.-C., and Strecker, G. (1977). Sialidosis (mucolipidosis I). Helvetica Paediatrica Acta, 32, 391-400.

Engel, J., Rapin, I., and Giblin, D. R. (1977). Electrophysiological studies in two patients with cherry red spot-myoclonus syndrome. Epilepsia, 18, 73-87.

Goldstein, M. L., Kolodny, E. H., Gascon, G. G., and Gilles, F. H. (1974). Macular cherry red spot, myoclonic epilepsy and neurovisceral storage disease in a 17-year-old girl. Transactions of the American Neurological Association, 99, 110-112.

Haldane, J. B. S. (1941). The relative importance of principal and modifying genes in determining some human disease. Journal of Genetics, 41, 149-157.

Itoyama, Y., Goto, I., Kuroiwa, Y., Takeuchi, M., Kawabuchi, M., and Tanaka, Y. (1978). Familial 
juvenile storage disease. Archives of Neurology (Chicago), 35, 792-800.

Johnson, W. G., and Chutorian, A. M. (1978). Inheritance of the enzyme defect in a new hexosaminidase deficiency disease. Annals of Neurology, 4, 399-413.

Johnson, W. G., Chutorian, A. M., and Miranda, A. (1977). A new juvenile hexosaminidase deficiency disease presenting as cerebellar ataxia: clinical and biochemical studies. Neurology (Minneapolis), 27, 1012-1018.

Justice, R. M., Wenger, D. A., Naidu, S., and Rosenthal, I. M. (1977). Enzymatic studies in a new variant of $\mathbf{G M}_{1}$ gangliosidosis in an older child. Paediatric Research, 11, 407.

Lowden, J. A., and O'Brien, J. S. (1979). Sialidosis: a review of human neuraminidase deficiency. American Journal of Human Genetics, 31, 1-18.

O'Brien, J. S. (1977). Neuraminidase deficiency in the cherry red spot-myoclonus syndrome. Biochemical and Biophysical Research Communications, 79, 1136-1141.

O'Brien, J. S. (1978). The cherry red spot-myoclonus syndrome: a newly recognized inherited lysosomal storage disease due to acid neuraminidase deficiency. Clinical Genetics, 14, 55-60.

O'Brien, J. S., and Rapin, I. (1977). Two sisters with the cherry red spot-myoclonus syndrome and oligosacchariduria. American Journal of Human Genetics, 29, 83A.

Rapin, I. S., Goldfischer, A., Katzman, R., Engel, J. Jr., and O'Brien, J. S. (1978). The cherry red spot-myoclonus syndrome. Annals of Neurology, 3, 224-242.

Rapin, I., Katzman, R., and Engel, J. Jr. (1975). Cherry red spots and progressive myoclonus without dementia: a distinct syndrome with neuronal storage. Transactions of the American Neurological Association, 100, 39-42.

Strecker, G., Hondi-Assah, T., Fournet, B., Spik, G., Montreuil, J., Maroteaux, P., Durand, P., and Farriaux, J. P. (1976). Structure of three major sialy-oligosaccharides excreted in the urine of three patients with three distinct inborn diseases. "I-cell disease" and two new types of mucolipidosis. Biochimica et Biophysica Acta (Amsterdam), 444, 349.

Strecker, G., Peers, M.-C., Michalski, J. C., HondiAssah, T., Fournet, B., Spik, G., Montreuil, J., Farriaux, J.-P., Maroteaux, P., and Durand, $\mathbf{P}$. (1977). Structure of nine sialyl-oligosaccharides accumulated in urine of eleven patients with three different types of sialidosis: mucolipidosis II and two new types of mucolipidosis. European Journal of Biochemistry, 75, 391-403.

Swallow, Dallas, Evans, Lorraine, Stewart, G., Thomas, P. K., and Abrams, J. D. (1979). Sialidosis type I: cherry red spot-myoclonus syndrome with sialidase deficiency and altered electrophoretic mobility of some enzymes known to be glycoproteins. II. Enzymes studies. Annals of Human Genetics, 43, 27-35.

Thomas, G. H., Tipton, R. E., Ch'Jen, L. T. Reynolds, L. W., and Miller, C. S. (1978). Sialidase ( $\alpha$-N-acetyl neuraminidase) deficiency: enzyme de्षे fect in an adult with macular cherry-red spots an myoclonus without dementia. Clinical Genetics, 13 369-379.

Wenger, D. A., Tarby, T. J., and Wharton, C. (1978) Macular cherry red spots and myoclonus with de mentia: coexistent neuraminidase and $\beta$-galactos dase deficiencies. Biochemical and Biophysical Reg search Communications, 82, 589-595. 